\title{
Experimental Study on the Improvement of the Thermal Behavior of Electric Motors
}

\author{
M.R. Guechi ${ }^{a}$, P. Desevaux ${ }^{a} *$ P. Baucour ${ }^{a}$, C. $_{\text {Espanet }}{ }^{a}$, R. Brunel ${ }^{b}$ and M. Poirot ${ }^{b}$ \\ ${ }^{a}$ University of Franche-Comté, FEMTO-ST-UMR CNRS 6174, Dept Energy, Belfort F-90000, France \\ ${ }^{b}$ INSA Lyon, IMP-UMR CNRS 5223, Villeurbanne F-69621, France
}

\begin{abstract}
The objective of this work is the improvement of the heat dissipation and the cooling of electric motors intended for the traction of vehicles. Two ways of improvement are proposed. The first one consists in replacing the air surrounding the stator windings by resins of higher thermal conductivity. The second way consists in using the spray cooling technique to cool the hottest parts of electric motor, i.e. coil windings. The two solutions are tested independently and simultaneously. Results show that filling the coil windings with resin enables better heat dissipation in the case of basic cooling by free air convection. On the contrary, the lowest temperatures are obtained with traditional coil winding without resin and by using spray cooling. In that case, the liquid part of the spray may infiltrate inside the coil and the evaporation takes place at the hottest point.
\end{abstract}

Keywords: Electric motor, Thermal performance, Heat dissipation, Windings impregnation, Spray cooling

\section{Introduction}

The work presented in this paper has been achieved in the framework of a research project aiming at improving the heat dissipation and the cooling of electric motors intended for new generations of electric cars (power higher than $30 \mathrm{~kW}$ ). Taking into account both specific power and torque expected for this new generation of vehicles, it is crucial to be able to evacuate efficiently the heat generated by the losses in the motor.

The basic cooling of the electric motors is generally obtained by natural or forced air convection modes [1, 2]. Unfortunately, surfaces inside the machine, which can be used for heat removal, have usually limited area and heat-transfer coefficients on these surfaces are relatively small. Due to high specific heat capacity of water and high liquid heat-transfer coefficients, substantial improvement can be achieved by liquid cooling. In this way, water jackets of many design types (axial and circumferential ducts) are used to allow the water to flow inside the stator and the rotor $[3,4]$.

${ }^{*}$ Corresponding author. Tel.: +33(0)384578225

Fax: +33(0)384570032; E-mail: philippe.desevaux@univ-fcomte.fr

(C) 2015 International Association for Sharing Knowledge and Sustainability

DOI: $10.5383 /$ ijtee.09.02.006
Liquid immersion is another cooling solution. For many years, the immerging evaporative cooling technique was successfully used in electrical machines $[5,6]$. This method has perfect cooling capability and high reliability, but it has the inconvenience of requiring a very high quantity of coolant.

The present work proposes to investigate two ways to improve the thermal behavior of an electric motor. The first way consists in improving the heat dissipation within the electric motor and concerns mainly the choice of materials constituting various parts of the engine $[7,8]$. The second way consists of the cooling method by pulverization of a liquid spray. The spray cooling technique has been previously used for cooling electronic components $[9,10]$ or for metal quenching $[11,12]$. Spray cooling is considered as one of the most promising techniques for the high power thermal management due to the advantages of high cooling efficiency, low mass flow rate and uniform cooling surface temperature distribution [13-15].

Different fluids may be used in spray cooling application. An oil spray cooling system for in-wheel motor in electric vehicles was developed in a recent study [16]. Oil-cooled mode has advantage that it can cool and lubricate at the same time but has lower cooling capacity than the water-cooled mode. To 
take the best advantage of the latent heat of phase change, it may be preferable to use fluids such as water or FC72 $[17,18]$. A numerical approach by Computational Fluid Dynamics has been previously carried out by the present authors $[19,20]$ in order to evaluate the efficiency of the spray cooling and to size and optimize the cooling system. For that, they have firstly developed a numerical model making it possible to predict the heat transfer with phase change between a heated surface and a two-phase impinging jet.

In this paper, an experimental approach of water spray cooling dedicated to cool the high temperature parts of an electrical motor is tested on a simple coil (a concentrated winding around one laminated tooth). The improvement way of the heat dissipation inside the coil consists in replacing the air surrounding the stator windings by resins of higher thermal conductivity. This paper is organized as follows. The next section presents the methodology used to improve the heat dissipation inside an electric motor and in particular the used impregnating resins. The third section describes the spray cooling setup and its instrumentation. Finally, the last section discusses the results obtained using the two improvement solutions independently and simultaneously.

\section{Heat Dissipation Improvement: Windings Impregnation with Resins}

A way to improve the dissipation of the heat produced by Joule effect within the coil windings consists in increasing thermal conductivity within the coil windings. For that, a well-known solution is impregnate the stator coil windings with resin which thus replaces the stagnant air present in traditional windings, as shown in Figure 1. Then, the difficulty lies in the choice of the optimal material of impregnation which must have both good electrical insulation properties and good thermal conductivity to facilitate the dissipation of the heat generated in the coil windings [21].

During this study, we have investigated classical and alternative resins; three different thermosetting resins (polyester and epoxy) with different thermal conductivities (Table 1) have been used to impregnate a stator coil winding (Figure 2) :

- The polyester resin Voltatex 4200, commercialized by DuPont. which is a low emission, environmentally friendly, yellowish $1 \mathrm{~K}$ dip resin based on unsaturated polyesterimide resins. This resin, which has excellent thermal and mechanical strength with outstanding longterm stress endurance, is commonly used to encapsulate electric and electronic components.

- The epoxy resin Damival 15244, commercialized by Von Roll. This resin, which has a good resistance to vibrations and a good thermal conductivity, is used as electrical insulating material.

- The third resin tested (i.e. Epoxy $+10 \%$ AlN) is a composite material prepared in laboratory. The base polymer used is the epoxy resin bisphenol-Aepichlorohydrin DGEBA-LY556 from Huntsman synthesized with a curing agent (diamine diphenyl sulfone). In order to improve the natural low thermal conductivity of epoxy resin, thermally conducting but electrically insulating semiconductor fillers (i.e. aluminum nitrides AlN) were used to dope the polymer matrix. The content of AlN in the resin used in the present study was $10 \%$ by volume.
The working temperature of the three tested resins should not exceed $180^{\circ} \mathrm{C}$.

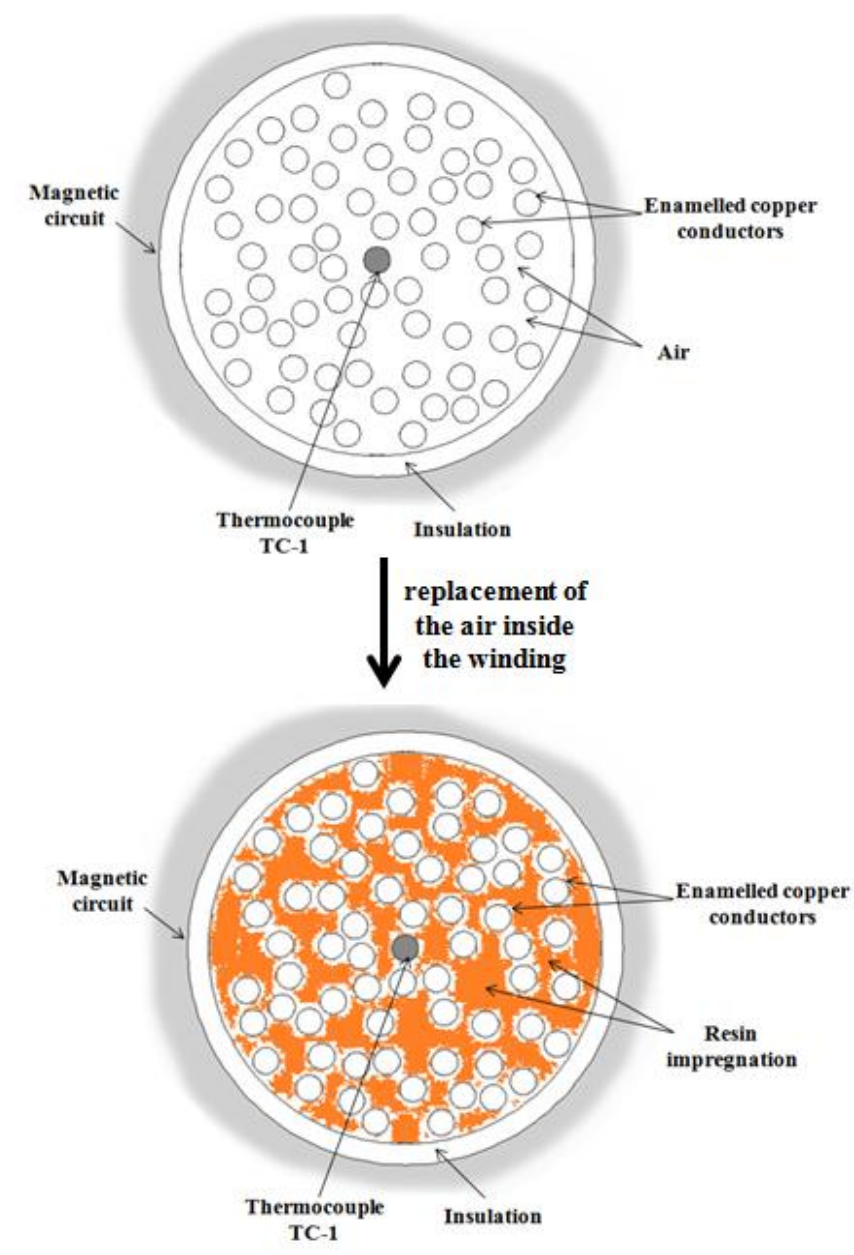

Fig.1. Principle scheme of a coil winding impregnation

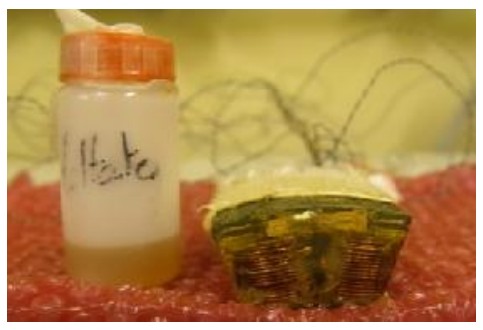

Fig.2. Coil winding filled with impregnating resin

Table 1 : Thermal conductivities of the tested resins

\begin{tabular}{cccc}
\hline Resins & $\begin{array}{c}\text { Voltatex 4200 } \\
\text { DuPont }\end{array}$ & $\begin{array}{c}\text { Epoxy }+10 \% \\
\text { AlN }\end{array}$ & $\begin{array}{c}\text { Damival } \\
15244 \\
\text { Von Roll } \\
\text { Epoxy }\end{array}$ \\
$\begin{array}{c}\text { Chemical } \\
\text { group }\end{array}$ & Polyester & Epoxy & 0.84 \\
$\begin{array}{c}\text { Thermal } \\
\text { conductivity } \\
\left(\mathrm{W} \cdot \mathrm{m}^{-1} \cdot \mathrm{K}^{-1}\right)\end{array}$ & 0.23 & 0.38 & \\
\hline
\end{tabular}




\section{Spray Cooling}

Spray cooling generally consists of a stream of fluid droplets (mixture of gas and fine liquid droplets), atomized through a nozzle and which impacts on the surface to be cooled. The droplets spread on the hot surface and can evaporate by nucleate boiling or form a thin liquid film, removing large amounts of energy at low temperatures. The mechanisms by which heat is removed during spray cooling involve the forced convection, thin liquid film evaporation, nucleate boiling and secondary nucleation. Further, this mechanically driven evaporation/condensation process is nearly isothermal and can effectively maintain components at quite uniform temperatures to improve reliability. In this work, the spray cooling technique is used to cool coil windings of an electric motor. The test bench designed to enable the experimental investigation of this cooling process is shown in figure 3 .

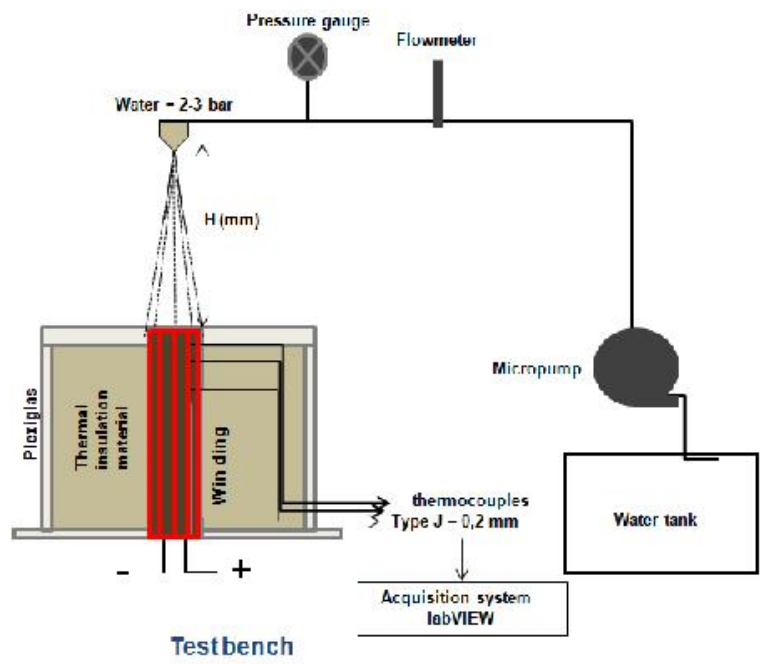

Fig.3. Spray cooling test bench

It consists of a heating system, a spraying system and a data acquisition system. The spraying surface corresponds to the top surface of a coil winding. The coil winding is supplied with a current intensity of $20 \mathrm{~A}$ and a voltage of $8 \mathrm{~V}$, corresponding to a current density of $20.31 \mathrm{~A} / \mathrm{mm}^{2}$ and copper losses of $160 \mathrm{~W}$. The current density is high according to the background of this study which deals with the cooling of a motor with high specific torque. The winding (except the top surface) is wrapped by thermal insulation material to minimize heat loss and to guarantee unidirectional heat conduction (in the axial direction).

The wound teeth used in the present experiments are identical to those used by the company Phenix International for the manufacture of radial flux permanent magnet motors of $7.6 \mathrm{~kW}$ power, wich is specially designed for a small urban electric vehicle.

A spray nozzle (Fine Spray Hydraulic Atomizing-type1/4MSS2 - spraying system) with a spray angle of $70^{\circ}$ and an orifice diameter of $0.71 \mathrm{~mm}$ is used. The spray nozzle is supplied with water at the operating pressure by means of a micro pump. As specified by the manufacturer, the spray nozzle used produces water droplets of about $214 \mu \mathrm{m}$ Sauter Mean Diameter at a supply pressure of 3 bar.

A flow meter (Brooks Instruments - R6-15B) and a Bourdon manometer are used to measure the flow rate and the inlet pressure of water, respectively. J-type (iron-constantan) thermocouples, each having a $0.2 \mathrm{~mm}$ bead diameter, were implanted at different positions as shown in figure 4.
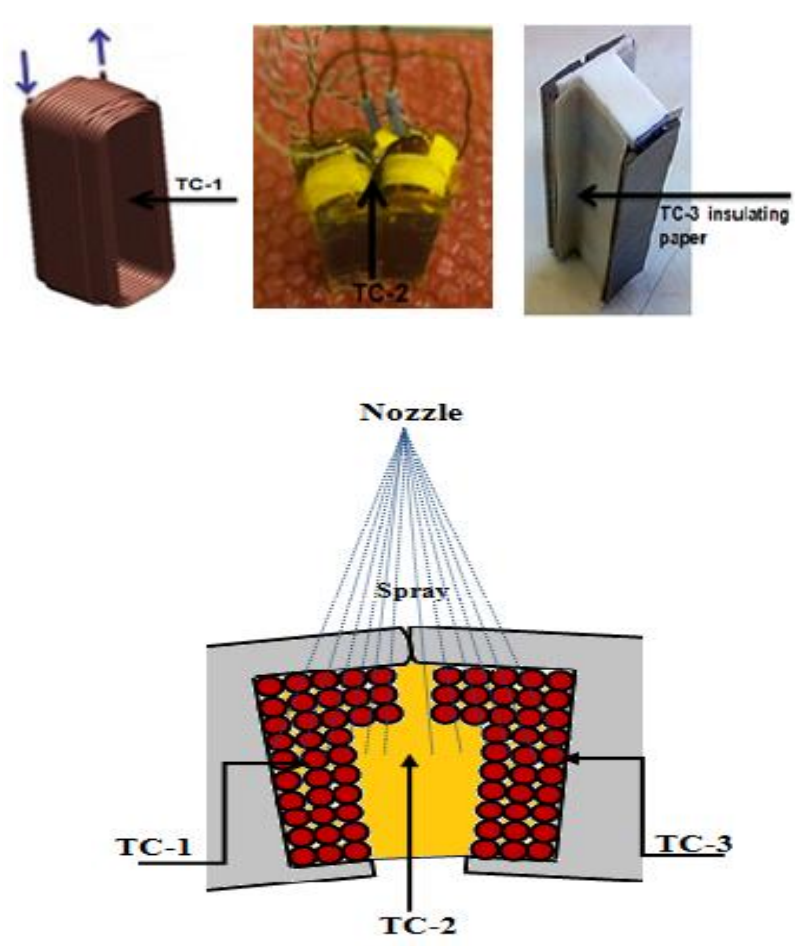

Fig. 4. Thermocouples position

The first thermocouple (TC-1) is positioned in the coil winding medium. The second thermocouple (TC-2) is positioned between two coils. The third thermocouple (TC-3) is located between the tooth and insulating paper medium. The thermocouple signals are collected using a high-density thermocouple module (NI9213 module) connected to a PC running with LabVIEW.

During the present experiments, the spray nozzle is positioned above and perpendicular to the horizontally placed heated surface. The water flow rate lies between $106 \mathrm{ml} / \mathrm{min}$ and 152 $\mathrm{ml} / \mathrm{min}$. The supply pressure of water is comprised between 2 and 4 bar and the water inlet temperature is fixed at $30^{\circ} \mathrm{C}$ or $40^{\circ} \mathrm{C}$. The winding temperature is carefully controlled in order not to exceed the temperature limit of the resin (approximately $\left.180^{\circ} \mathrm{C}\right)$.

\section{Results and Discussion}

This section presents experimental results obtained using traditional and impregnated coil windings, cooled by free air convection or by water spray.

Figures 5 and 6 compare the evolutions of the temperature inside a traditional coil winding and coil windings filled with impregnating resins. 


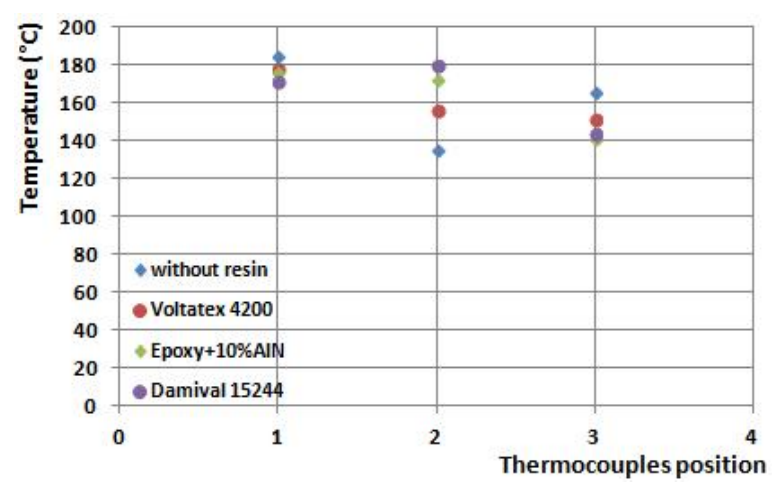

Fig. 5. Evolution of the temperature inside the coil winding - Free air convection

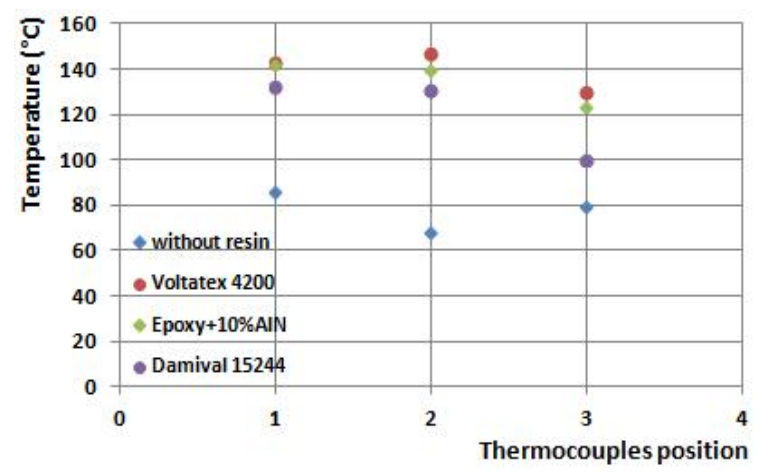

Fig. 6. Evolution of the temperature inside the coil winding Spray cooling

The results obtained when the coil winding is cooled by free air convection only (Figure 5) show a non-monotonic evolution of the temperature inside the traditional coil winding (without impregnating resin). The lowest temperature is measured between the two teeth. When replacing air by resin, the temperatures TC- 1 and TC- 3 decrease but the temperature between the two teeth is higher than without impregnating resin. This is explained by the fact that the resin, which is a better heat conductor than air, improves the heat transfer by conduction from the coil winding medium and the teeth iron medium. The temperatures $\mathrm{TC}-1$ and $\mathrm{TC}-3$ are thus logically lower than those measured without resin. On the other hand, the thermocouple TC-2 indicates the temperature between the teeth. When the impregnating resin replaces the stagnant air, the gain in thermal conductivity leads to an increase of the temperature at this place.

This phenomenon may also be seen in figure 6 in the case of spray cooling of the coil winding (water flow rate: $132 \mathrm{ml} / \mathrm{min}$, water temperature: $40^{\circ} \mathrm{C}$ ). Moreover, it appears clearly that the presence of resin is penalizing when using this mode of cooling. Indeed, the lowest temperatures are obtained in the traditional coil winding. This is explained by the fact that the upper surface of the impregnated coil winding is covered by a thin layer of resin which slows down the convective heat exchange at the surface. This limitation is all the more significant that the mode of cooling is effective and that the thermal conductivity of the resin is low. Furthermore, when no resin is used, the liquid part of the spray may infiltrate inside the coil and the evaporation which takes place at the hottest point increases the heat removal capability of the spray.
Figures 7 and 8 show the effect of the spray cooling on the thermal behavior of the coil winding and in particular the influence of the spray flow rate used.

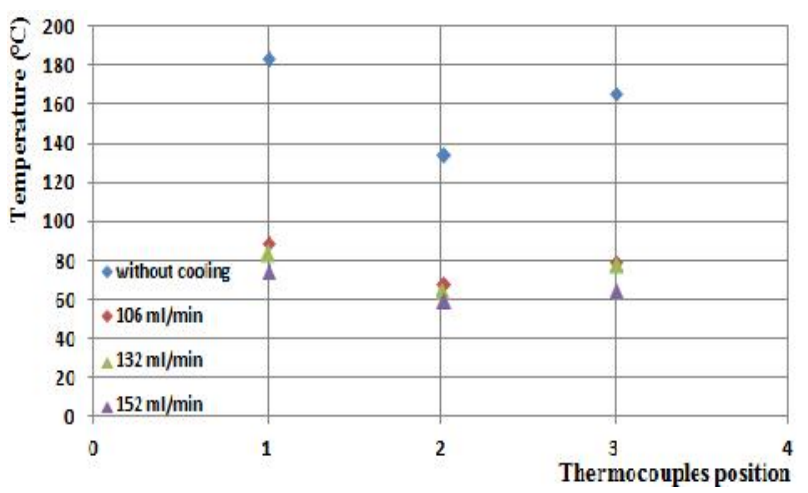

Fig. 7. Evolution of the temperature inside the coil winding for different spray flow rates - Coil winding without resin

Varying the input flow rate is obtained by varying the supply pressure of the spray nozzle between 2 and 4 bar.

The results obtained in the case of traditional coil windings (without impregnating resin) are presented in figure 7 . They show a clear reduction in the temperatures inside the coil windings thanks to spray vaporization. This reduction, which increases with increasing the spray flow rate, is less marked when the air is replaced by impregnating resin, as shown in figure 8 .

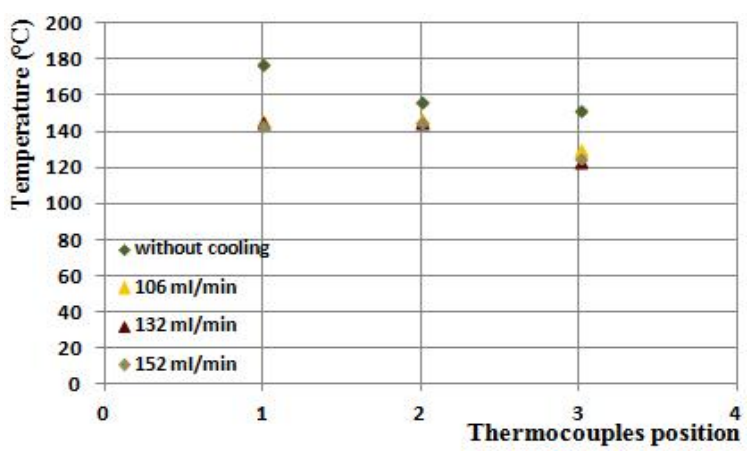

Fig. 8. Evolution of the temperature inside the coil winding for different spray flow rates - Coil winding filled with resin Voltatex 4200

Figure 9 illustrates the evolution of the cooling performance with the spray flow rate, in the case of coil windings impregnated with resin Voltatex 4200. The temperatures measured by the 3 thermocouples show a clear improvement of the cooling performance when the flow rate increases till a value between 132 and $152 \mathrm{ml} / \mathrm{min}$. An approximately $5^{\circ} \mathrm{C}$ reduction of the coil winding temperatures is observed. This is due to the fact that increasing the liquid flow rate leads to an increase of the nozzle inlet pressure. Therefore, droplets that are delivered to the impact surface have a smaller diameter and a higher velocity. That results in an intensification of the forced convection at the surface to be cooled. For higher values of flow rate, a degradation of the cooling performance is observed. This degradation may be explained by the fact that the angle of spray produced by the spray nozzle increases with the spray flow rate. Consequently, for larger spray flow rate, 
some liquid sprays outside the test surface. The effective flow covering the test surface is reduced and the cooling performance is therefore degraded.

Finally, these results seem to highlight the existence of an optimal value of spray flow rate (comprise between 132 and $152 \mathrm{ml} / \mathrm{min}$ for our current experimental setup).

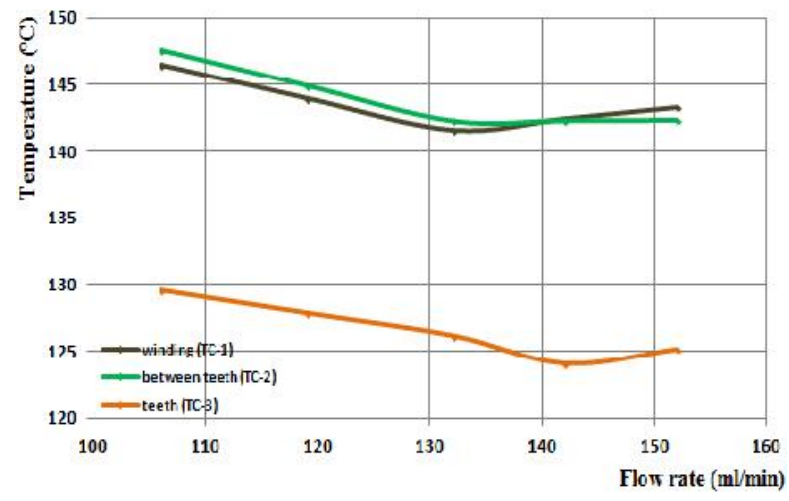

Fig. 9. Effect of spray flow rate on the coil temperature - Coil winding filled with resin Voltatex 4200

Figures 10 and 11 present the results obtained for 4 values of power (between 115 and $160 \mathrm{~W}$ ) injected in the windings.

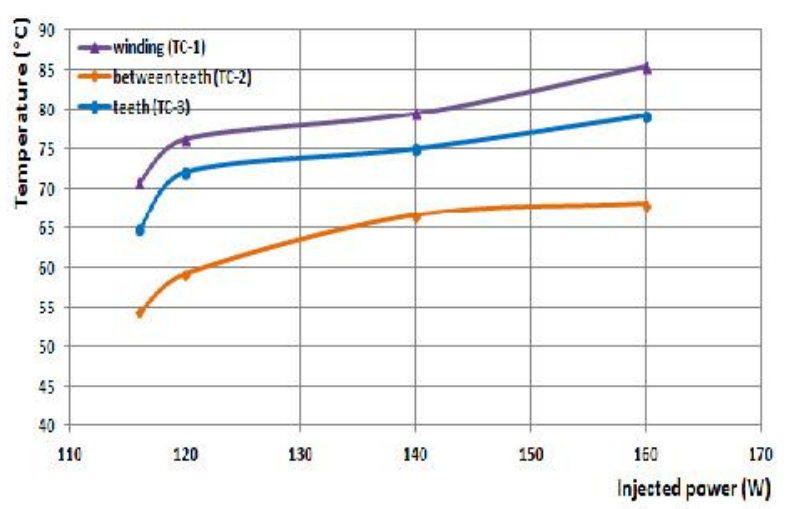

Fig. 10. Evolution of the temperatures inside the coil winding with the injected power - Coil winding without resin

The tests have been carried out using spray cooling (water flow rate: $132 \mathrm{ml} / \mathrm{min}$, water temperature: $40^{\circ} \mathrm{C}$ ). The evolutions of temperature obtained with a traditional winding (without impregnating resin) are comparable with those obtained with the winding impregnated with resin Voltatex 4200. The temperatures inside the windings increase significantly with the injected power. It is observed that, when using spray cooling, the levels of temperature reached in the winding with resin Voltatex 4200 are definitely higher than those raised in the traditional winding. Indeed, the recorded maximum temperature does not exceed $85^{\circ} \mathrm{C}$ in the traditional winding whereas it reaches $145^{\circ} \mathrm{C}$ in the impregnated winding.

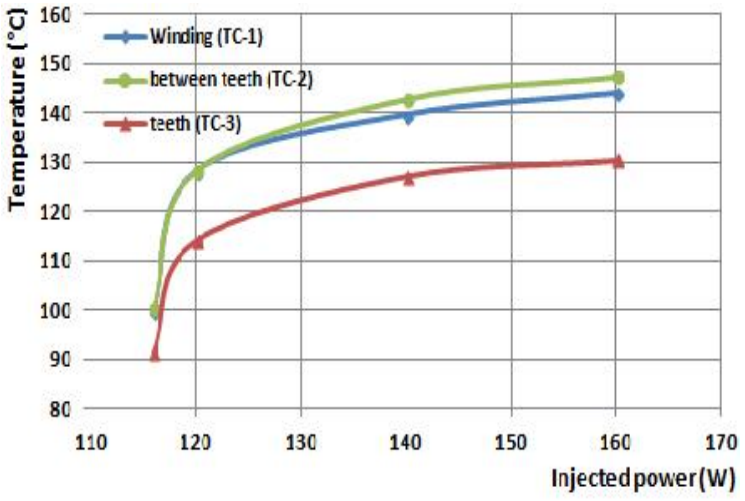

Fig. 11. Evolution of the temperatures inside the coil winding with the injected power - Coil winding filled with resin Voltatex 4200

Figures 12 and 13 show the effect of the input fluid temperature on the spray cooling performance. Experiments have been carried out with the traditional coil winding (figure 12 ) and the coil winding filled with resin Voltatex 4200 (figure 13), by using input water at temperature of $30^{\circ} \mathrm{C}$ and $40^{\circ} \mathrm{C}$ (water flow rate of $132 \mathrm{ml} / \mathrm{min}$ ).

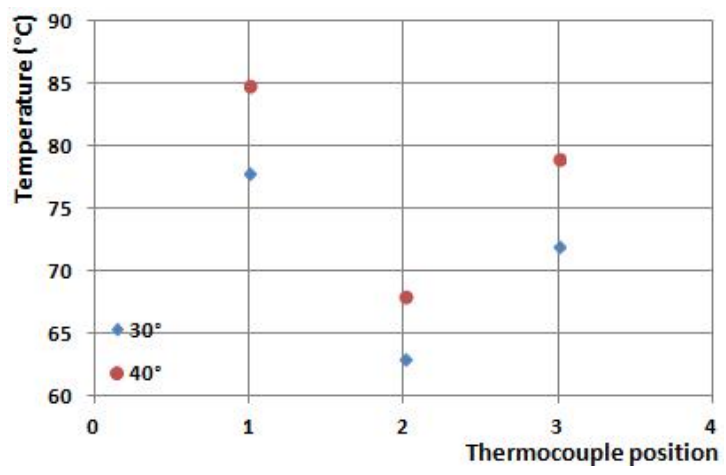

Fig. 12. Evolution of the temperatures inside the coil winding for different values of the input fluid temperature - Coil winding without resin

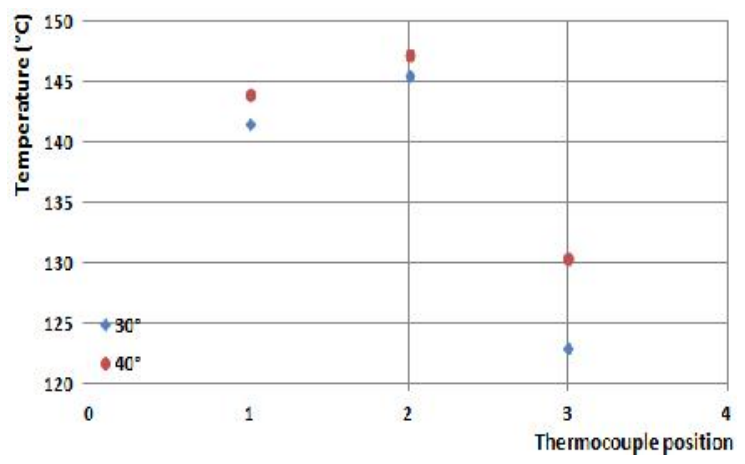

Fig. 13. Evolution of the temperatures inside the coil winding for different values of the input fluid temperature - Coil winding filled with resin Voltatex 4200 
It may be observed a significant influence of the spray inlet temperature on the cooling of both types of the windings, even if it is more marked for the traditional winding without resin. For this winding, one observes a reduction in the temperature that can reach $7^{\circ} \mathrm{C}$ in certain places of the winding when decreasing by $10^{\circ} \mathrm{C}$ the inlet temperature of the spray.

\section{Conclusion}

This paper has presented two ways to improve the thermal behavior of an electric motor. The first way consists in improving the heat dissipation within the electric motor by replacing the air surrounding the stator windings by special resins of higher thermal conductivity. The second way consists in using the water spray cooling technique to cool the hottest parts (i.e. coil windings) of an electric motor. One originality of this work lies in the combination of these two solutions techniques that have been tested independently and simultaneously.

The tests carried out for different values of power injected in coil windings and for different temperatures of input spray water have highlighted the interest of the cooling spray technique. In all the tested cases, the levels of temperature reached in the coil windings are lower than those obtained with free convection cooling. A significant influence of the inlet temperature of the spray water on the cooling performance has been observed.

The interest to fill coil windings with impregnating resin of good thermal conductivity is less obvious. When using spray cooling, the lowest temperatures are reached within traditional coil windings. On the other hand, in the case of a traditional cooling by free convection, the replacement of the air by resin may be interesting by limiting temperatures in the coil windings.

A comparison between the heat transfer with and without impregnating resin in the case of a cooling by forced air convection is currently underway. The replacement of water by another dielectric liquid (FC-72 of HFE-7100) is also under study.

The whole of these experimental results, supplemented by CFD simulations of the spray cooling, will enable to design the cooling system before its implementation on an electric motor test bench.

\section{References}

[1] Staton D.A., Cavagnino A. Convection heat transfer and flow calculations suitable for electric machines thermal models, Industrial Electronics, IEEE Transactions on, 2008, 55, 3509-3516.

[2] Farsane K., Desevaux P., Panday P.K. Experimental study of the cooling of a closed type electric motor, Applied Thermal Engineering, 2000, 20, 1321-1334.

[3] Boglietti A., Staton D.A., Cavagnino A., Shanel M., Mueller M., Mejuto C. Evolution and modern approaches for thermal analysis of electrical machines, Industrial Electronics, IEEE Transactions on, 2009, 56, 871-882.

[4] Kulkarni D.P. Experimental investigation of contact resistance for water cooled jacket for electric motors and generators, IEEE Transactions on Energy Conversion, 2012, 27, 204-201.
[5] Wen Z.W, Gu G.B, Wang H.F. Calculation of 3D thermal field in the stator of turbo- generator with immersion evaporative cooling system and forced inner-cooling, Proceedings of the CSEE, 2006, 26, 133-138.

[6] Luan R., Fu D.P., Tang L.Y. Study on 3D temperature distribution in new evaporative cooling asynchronous generator, Proceedings of the CSEE, 2004, 24, 205-209.

[7] Simpson N., Wrobel R., Mellor P. Estimation of Equivalent Thermal Parameters of Impregnated Electrical Windings, IEEE Transactions on Industry Applications, 2013, 49, 2505-2515.

[8] Tsukamoto O., Ishigohka T., Yamamoto M., Tanaka Y., Kobayashi H. Characteristics of epoxyimpregnated AC superconducting winding, IEEE Transactions on Magnetics, 1987, 23, 1592-1595.

[9] Hsieh C.C, Yao S.C. Evaporative heat transfer characteristics of a water spray on micro-structured silicon surfaces, International Journal of Heat and Mass Transfer, 2006, 49, 962-974.

[10] Sodtke C., Stephan P. Spray cooling on micro structured surfaces, International Journal of heat and Mass Transfer, 2007, 50, pp. 4089-4097.

[11] Hall D.D, Mudawar I. Experimental and numerical study of quenching complex-shaped metallic alloys with multiple overlapping sprays, International Journal of Heat and Mass Transfer, 1995, 38, 12011216.

[12] Puschmann F., Specht E., Schmidt J. Local distribution of the heat transfer in water spray quenching, International Conference on Continuous Casting of Non-Ferrous Metals. Proceedings of the DGM, 2000, Frankfurt, 101-107.

[13] Kim J. Spray cooling heat transfer : the state of the art, International Journal of Heat and Fluid Flow, 2007, 28, 753-767.

[14] Visaria M., Mudawar I. Application of two-phase spray cooling for thermal management of electronic devices, IEEE Trans. Compon. Pack. Technol., 2009, 32, 784-793.

[15] Hou Y., Liu X., Liu J., Li M., Pu L. Experimental study on phase change spray cooling, Experimental Thermal and Fluid Science, 2013, 46, 84-88.

[16] Lim D.H, Kim S.C. Thermal performance of oil spray cooling system for in-wheel motor in electric vehicles, Applied Thermal Engineering, 2014, 63, 577-587.

[17] Lin L., Ponnappan R. Heat transfer charcateristics of spray cooling in a closed loop, International Journal of Heat and Mass Transfer, 2003, 46, 3737-3746.

[18] Pautsch A.G. Heat transfer and film thickness characteristics of spray cooling with phase change, MS Thesis, University of Wisconsin-Madison, 2004.

[19] Guechi M., Desevaux P., Baucour P. Numerical and experimental study of spray cooling of a heated metal surface, ICLASS 2012, $12^{\text {th }}$ Triennial International Conference on Liquid Atomization and Spray Systems, September 2012. 
[20] Guechi M., Desevaux P, Baucour P. On the numerical and experimental study of spray cooling, The Journal of Computational Multiphase Flows, 2013, 5, 239-249.

[21] Kochetov R., Andritsch T., Lafont U., Morshuis P.H.F., Picken S.J., Smit J.J. Thermal Behaviour of
Epoxy Resin Filled with High Thermal Conductivity nanopowders, Proceedings of IEEE Electrical Insulation Conference, 2009, Montreal, QC, Canada, 524-528. 